\title{
SOME SHARP NEIGHBORHOODS OF UNIVALENT FUNCTIONS
}

\author{
BY
}

\author{
JOHNNY E. BROWN
}

\begin{abstract}
For $\delta \geqslant 0$ and $f(z)=z+a_{2} z^{2}+\cdots$ analytic in $|z|<1$ let the $\delta$ neighborhood of $f, N_{\delta}(f)$, consist of those analytic functions $g(z)=z+b_{2} z^{2}+\cdots$ with $\sum_{k=2}^{\infty} k\left|a_{k}-b_{k}\right| \leqslant \delta$. We determine sufficient conditions guaranteeing which neighborhoods of certain classes of convex functions belong to certain classes of starlike functions. We extend some recent results of St. Ruscheweyh and R. Fournier and, at the same time, provide much simpler proofs. We also prove precisely how boundaries affect the value of $\delta$ for some general classes of functions.
\end{abstract}

Let $\mathscr{H}$ denote the class of functions analytic in the unit disk $\mathbf{D}$ normalized so that $f(0)=0$ and $f^{\prime}(0)=1$. If $f(z)=z+a_{2} z^{2}+\cdots \in \mathscr{H}$ and $\delta \geqslant 0$ we define the $\delta$-neighborhood of $f$ by

$$
N_{\delta}(f)=\left\{g(z)=z+b_{2} z^{2}+\cdots \in \mathscr{H}: \sum_{k=2}^{\infty} k\left|a_{k}-b_{k}\right| \leqslant \delta\right\} .
$$

A. W. Goodman [3] proved that if $f_{0}(z)=z$, the identity function in $\mathscr{H}$, then $N_{1}\left(f_{0}\right) \subset S^{*}$, the class of starlike functions in $\mathscr{H}$. St. Ruscheweyh [5] recently extended this result and proved that if $f(z)=z+a_{n+1} z^{n+1}+\cdots \in C$ (convex functions in $\mathscr{H}$ ), then $N_{\delta_{n}}(f) \subset S^{*}$ for $\delta_{n}=2^{-2 / n}$. In the same paper he asked whether a corresponding result would hold if $C$ were replaced by $\tilde{T}$ (functions in $\mathscr{H}$ with $\left|z f^{\prime \prime}(z) / f^{\prime}(z)\right|<1$ ) and $S^{*}$ replaced by $T$ (functions in $\mathscr{H}$ with $\left|z f^{\prime}(z) / f(z)-1\right|$ $<1)$. R. Fournier [2] has now proved the corresponding result for $\tilde{T}$ and $T$. The purpose of this paper is to prove an analogous result for a specific one-parameter family of functions in $\mathscr{H}$ which will contain all of the above results as special cases. It should be noted that St. Ruscheweyh used results related to the Pólya-Schoenberg conjecture (established in [6]) as well as an extended version of the Clunie-Jack Lemma. We show that none of this machinery is necessary. The results obtained are best possible.

St. Ruscheweyh also proved that if $f \in S_{\alpha}^{*}$ (starlike functions of order $\alpha$ ), then there is no value of $\delta>0$ such that $N_{\delta}\left(S_{\alpha}^{*}\right) \subset S^{*}$ for any $0 \leqslant \alpha<1$. On the other hand, R. Fournier considers a subclass $T_{\alpha}$ of $T$ for which there exists a (sharp) $\delta>0$ with $N_{\delta}\left(T_{\alpha}\right) \subset T$. The differences in the two cases reduce to a question of boundaries.

Received by the editors June 15, 1983.

1980 Mathematics Subject Classification. Primary 30C45, 30C80; Secondary 30C75.

Key words and phrases. Univalent functions, Hadamard product, subordination, starlike functions, convex functions. 
We show precisely how the boundaries influence the value of $\delta$ for more general (but related) classes of functions.

Main results. Let $0 \leqslant B \leqslant 1$ and define the class $S^{*}(B)$ to be those functions $f \in \mathscr{H}$ for which

$$
\frac{z f^{\prime}(z)}{f(z)} \prec \frac{1+z}{1-B z}, \quad z \in \mathbf{D},
$$

where $\prec$ denotes subordination (i.e., $g \prec h$ if $g(z)=h(\omega(z))$, where $\omega(0)=0$ and $|\omega| \leqslant 1)$. Let $C(B)$ be those functions $f \in \mathscr{H}$ such that

$$
1+\frac{z f^{\prime \prime}(z)}{f^{\prime}(z)} \prec \frac{1+z}{1-B z}, \quad z \in \mathbf{D} .
$$

We observe that $S^{*}(B) \subset S^{*}$ and $C(B) \subset C$ and note also that $S^{*}(0)=T$ and $C(0)=\tilde{T}$. Our main result can now be stated.

THEOREM 1. Let $0 \leqslant B \leqslant 1$. If $f(z)=z+a_{n+1} z^{n+1}+\cdots \in C(B)$, then $N_{\delta_{n}}(f)$ $\subset S^{*}(B)$, where

$$
\delta_{n}= \begin{cases}(1+B)^{-(1+B) / B n}, & B \neq 0 \\ e^{-1 / n}, & B=0\end{cases}
$$

This result is sharp.

It is clear that $B=0$ and $B=1$ give the results of $\mathrm{R}$. Fournier [2] and St. Ruscheweyh [5], respectively. From (1) we see that $f \in S^{*}(B)$ if and only if

$$
\frac{z f^{\prime}(z)}{f(z)} \neq \frac{1+e^{i \theta}}{1-B e^{i \theta}}, \quad 0 \leqslant \theta \leqslant 2 \pi .
$$

It follows that $f \in S^{*}(B)$ if and only if $h_{\theta}(z) * f(z) / z \neq 0, z \in \mathbf{D}$, where $*$ denotes the Hadamard product and $h_{\theta}(z)$ is defined as

$$
h_{\theta}(z)=(1+B)^{-1} e^{-i \theta}\left\{\frac{\left(1+e^{i \theta}\right) z}{(1-z)}-\frac{\left(1-B e^{i \theta}\right) z}{(1-z)^{2}}\right\} \text {. }
$$

If $h_{\theta}(z)=z+\sum_{k=2}^{\infty} C_{k} z^{k}$, then $C_{k}=\left[(1-k)+e^{i \theta}(1+B k)\right] / e^{i \theta}(1+B)$ and hence $\left|C_{k}\right| \leqslant k$. A sufficient condition for $N_{\delta}(f) \subset S^{*}(B)$ can now be given. Following [5] we suppose $g(z)=z+b_{2} z^{2}+\cdots \in N_{\delta}(f)$, where $f(z)=z+a_{2} z^{2}+\cdots \in \mathscr{H}$ and $\left|h_{\theta} * f / z\right| \geqslant \delta>0$. Then we have (since $\left|C_{k}\right| \leqslant k$ )

$$
\begin{aligned}
\left|\frac{h_{\theta} * g}{z}\right| & \geqslant\left|\frac{h_{\theta} * f}{z}\right|-\left|\frac{h_{\theta} *(g-f)}{z}\right| \\
& \geqslant \delta-\left|\sum_{k=2}^{\infty} C_{k}\left(a_{k}-b_{k}\right) z^{k}\right|>\delta-\sum_{k=2}^{\infty} k\left|a_{k}-b_{k}\right| \geqslant 0 .
\end{aligned}
$$

Hence $h_{\theta} * g / z \neq 0$ for $z \in \mathbf{D}$ so $g \in S^{*}(B)$. Hence to prove the theorem we need only prove that if $f(z)=z+a_{n+1} z^{n+1}+\cdots \in C(B)$, then

$$
\left|h_{\theta} * f / z\right| \geqslant \delta_{n}
$$

where $\delta_{n}$ is as defined in the theorem. We will need several preliminary lemmas. 
LEMMA 1. If $f(z)=z+a_{n+1} z^{n+1}+\cdots \in C(B)$, then

$$
\left|f^{\prime}(z)\right| \geqslant \begin{cases}\exp \left[-r^{n} / n\right], & B=0 \\ \left(1+B r^{n}\right)^{-(1+B) / B n}, & B \neq 0\end{cases}
$$

$|z|=r, 0 \leqslant r<1$.

Proof. It is easy to see that if $f(z)=z+a_{n+1} z^{n+1}+\cdots \in C(B)$, then by (2) we must have

$$
1+\frac{z f^{\prime \prime}(z)}{f^{\prime}(z)}=\frac{1+\omega(z)}{1-B \omega(z)}
$$

where $\omega(z)=z^{n} \phi(z)$ with $\phi(0) \neq 0$ and $|\phi(z)| \leqslant 1$. From (5) it follows that the range of values of $1+z f^{\prime \prime} / f^{\prime},|z| \leqslant r$, lies inside the disk $\Delta(r)$ given by

$$
\Delta(r)=\left\{w:\left|w-w_{0}\right| \leqslant R\right\},
$$

where $w_{0}=\left(1+B r^{2 n}\right) /\left(1-B^{2} r^{2 n}\right), R=(1+B) r^{n} /\left(1-B^{2} r^{2 n}\right)$ and $0 \leqslant r \leqslant 1$. It is then easy to check that

$$
1+\operatorname{Re}\left\{\frac{z f^{\prime \prime}(z)}{f^{\prime}(z)}\right\} \geqslant \frac{1-r^{n}}{1+B r^{n}}, \quad|z|=r .
$$

From this we observe that

$$
r \frac{\partial}{\partial r} \operatorname{Re}\left\{\log f^{\prime}\left(r e^{i \theta}\right)\right\}=\operatorname{Re}\left\{\frac{r e^{i \theta} f^{\prime \prime}\left(r e^{i \theta}\right)}{f^{\prime}\left(r e^{i \theta}\right)}\right\} \geqslant \frac{-(1+B) r^{n}}{1+B r^{n}}
$$

The result now follows by integration.

LEMMA 2. If $f(z)=z+a_{n+1} z^{n+1}+\cdots \in C(B)$, then $h_{\theta} * f$ is close-to-convex, hence univalent, for each $\theta \in[0,2 \pi)$.

Proof. If $\theta=0$ and $B=1$, then $h_{\theta}(z)=z /(1-z)$ and hence $h_{\theta} * f=f$. We may thus assume that $0<\theta<2 \pi$ or $0 \leqslant B<1$. From the definition of $h_{\theta}$ and properties of the Hadamard product we have

$$
\frac{e^{i \theta}(1+B)}{\left(1-B e^{i \theta}\right)} \frac{\left(h_{\theta} * f\right)^{\prime}}{f^{\prime}}=\left[\left(\frac{1+e^{i \theta}}{1-B e^{i \theta}}\right)-\left(1+\frac{z f^{\prime \prime}}{f^{\prime}}\right)\right]
$$

Let $\theta$ be fixed and put $\theta_{0}=\arg \left\{e^{i \theta} /\left(1-B e^{i \theta}\right)\right\}$. Then we see that for any $\alpha \in \mathbf{R}$

$$
e^{i\left(\theta_{0}+\alpha\right)} \frac{\left(h_{\theta} * f\right)^{\prime}}{f^{\prime}}=\frac{\left|1-B e^{i \theta}\right|}{(1+B)} e^{i \alpha}\left[\left(\frac{1+e^{i \theta}}{1-B e^{i \theta}}\right)-\left(1+z \frac{f^{\prime \prime}}{f^{\prime}}\right)\right] .
$$

Now from (6) it follows that the range of values of $1+z f^{\prime \prime} / f^{\prime}, z \in \mathbf{D}$, lies in the interior of $\Delta=\Delta(1)$. It is evident that the interior of $\Delta$ lies strictly inside the circle (or to the right of the line if $B=1$ )

$$
\Gamma: w(t)=\left(1+e^{i t}\right) /\left(1-B e^{i t}\right), \quad 0 \leqslant t<2 \pi .
$$

Now $\left(1+e^{i \theta}\right) /\left(1-B e^{i \theta}\right) \in \Gamma$ so we can choose $\alpha=\alpha(\theta)$ so that the right-hand side of (8) has positive real part. As $f \in C(B) \subset C$, we conclude that $h_{\theta} * f$ is close-to-convex (see [4]). 
LEMMA 3. If $f(z)=z+a_{n+1} z^{n+1}+\cdots \in C(B)$, then $\left|\left(1+e^{i \theta}\right)-\left(1-B e^{i \theta}\right)\left(1+\frac{z f^{\prime \prime}}{f^{\prime}}\right)\right| \geqslant(1+B)\left(\frac{1-r^{n}}{1+B r^{n}}\right), \quad|z|=r, 0 \leqslant \theta<2 \pi$.

Proof. The lemma is obviously true for $\theta=0$ and $B=1$. So suppose $0<\theta<2 \pi$ or $0 \leqslant B<1$ and define

$$
H(w)=\left(1-B e^{i \theta}\right)\left\{\left(\frac{1+e^{i \theta}}{1-B e^{i \theta}}\right)-w\right\}, \quad w \in \Delta(r),
$$

where $\Delta(r)$ is defined by (6). Now since $\Delta(r) \cap \Gamma=\varnothing, \Gamma$ defined by (9), we see that $H \neq 0$ on $\Delta(r)$. We can then apply the minimum principle to $\log |H(w)|$ to conclude that

$$
\begin{aligned}
|H(w)| & \geqslant \min _{w \in \partial \Delta(r)}|H(w)|=\min _{\substack{0<\theta<2 \pi \\
0 \leqslant \tau \leqslant 2 \pi}}\left|\left(1+e^{i \theta}\right)-\left(1-B e^{i \theta}\right)\left(\frac{1+r^{n} e^{i n \tau}}{1-B r^{n} e^{i n \tau}}\right)\right| \\
& =(1+B)\left(\frac{1-r^{n}}{1+B r^{n}}\right) .
\end{aligned}
$$

The result now follows.

LEMMA 4. Let

$$
\nu(r)=\frac{1}{r} \int_{0}^{r} \frac{1-t^{n}}{\left(1+B t^{n}\right)^{m+1}} d t, \quad m>0
$$

and

$$
\mu(r)=\frac{1}{r} \int_{0}^{r}\left(1-t^{n}\right) e^{-t^{n} / n} d t
$$

Then both are decreasing functions of $r$ for $0<r<1$.

Proof. Note that

$$
\nu^{\prime}(r)=-\frac{1}{r}\left[\nu(r)-\frac{\left(1-r^{n}\right)}{\left(1+B r^{n}\right)^{m+1}}\right], \quad 0<r<1 .
$$

Thus we clearly have $\nu^{\prime}(r)<0$ for $0<r<1$. To see this, fix $0<r<1$ and note by the mean value theorem it is enough to show that

$$
\beta(x) \equiv \frac{1-x^{n}}{\left(1+B x^{n}\right)^{m+1}} \geqslant \frac{1-r^{n}}{\left(1+B r^{n}\right)^{m+1}} \quad \text { for all } x \in(0, r) .
$$

Since $m>0, \beta(x)$ is decreasing and so $\nu^{\prime}<0$. The proof for $\mu(r)$ is similar and is omitted.

Proof of Theorem 1. Let $f(z)=z+a_{n+1} z^{n+1}+\cdots \in C(B)$. From (7) and Lemma 3 we see that

$$
\begin{aligned}
\left|\left(h_{\theta} * f\right)^{\prime}\right| & =\frac{\left|f^{\prime}(z)\right|}{(1+B)}\left|\left(1+e^{i \theta}\right)-\left(1-B e^{i \theta}\right)\left(1+z \frac{f^{\prime \prime}}{f^{\prime}}\right)\right| \\
& \geqslant\left|f^{\prime}(z)\right|\left(\frac{1-r^{n}}{1+B r^{n}}\right) .
\end{aligned}
$$


Now we apply Lemma 1 to obtain

$$
\left|\left(h_{\theta} * f\right)^{\prime}\right| \geqslant \begin{cases}\left(1-r^{n}\right) \exp \left\{-r^{n} / n\right\}, & B=0, \\ \frac{1-r^{n}}{\left(1+B r^{n}\right)^{((1+B) / B n)+1}}, & B \neq 0 .\end{cases}
$$

Fix $0<r<1$ and choose $z_{0},\left|z_{0}\right|=r$, so that $\left|\left(h_{\theta} * f\right)\left(z_{0}\right)\right|=\min _{|z|=r}\left|\left(h_{\theta} * f\right)(z)\right|$. By Lemma 2, $h_{\theta} * f$ is univalent and hence the preimage $L$ of the segment from 0 to $\left(h_{\theta} * f\right)\left(z_{0}\right)$ is an arc inside $|z| \leqslant r$. Thus we conclude that for $|z| \leqslant r$

$$
\begin{aligned}
\left|\left(h_{\theta} * f\right)(z)\right| & \geqslant\left|\left(h_{\theta} * f\right)\left(z_{0}\right)\right|=\int_{L}\left|\left(h_{\theta} * f\right)^{\prime}(z)\right||d z| \\
& \geqslant \int_{0}^{r}\left|\left(h_{\theta} * f\right)^{\prime}(z)\right| d r .
\end{aligned}
$$

Using (10) we conclude that

$$
\left|\frac{h_{\theta} * f}{z}\right| \geqslant \begin{cases}\mu(r), & B=0, \\ \nu(r), & B \neq 0,\end{cases}
$$

where $\mu, \nu$ are defined in Lemma 4 with $m=(1+B) / B n$. Applying that lemma we see that

$$
\left|\frac{h_{\theta} * f}{z}\right| \geqslant \begin{cases}\mu(1), & B=0 \\ \nu(1), & B \neq 0 .\end{cases}
$$

The right-hand side then determines $\delta_{n}$. To evaluate $\mu(1)$ and $\nu(1)$ we simply put

$$
I(z)= \begin{cases}z \exp \left\{-z^{n} / n\right\}, & B=0, \\ z\left(1+B z^{n}\right)^{-((1+B) / B n)}, & B \neq 0,\end{cases}
$$

and note that

$$
I^{\prime}(z)= \begin{cases}\left(1-z^{n}\right) e^{-z^{n} / n}, & B=0 \\ \frac{1-z^{n}}{\left(1+B z^{n}\right)^{m+1}}, & B \neq 0\end{cases}
$$

Thus $\mu(1)$ and $\nu(1)$ are now easily found. Hence $\delta_{n}$ is given as in the theorem.

To prove sharpness, we define $f(z)$ by

$$
f(z)= \begin{cases}\int_{0}^{z} e^{\xi^{n} / n} d \xi, & B=0 \\ \int_{0}^{z}\left(1-B \xi^{n}\right)^{-((1+B) / B n)} d \xi, & B \neq 0 .\end{cases}
$$

Clearly

$$
f(z)=z+a_{n+1} z^{n+1}+\cdots \in C(B) .
$$

If we let $g(z)=f(z)+\delta_{n} z^{n+1} /(n+1)$, then clearly $g \in N_{\delta_{n}}(f)$ and $g^{\prime}(z)=f^{\prime}(z)$ $+\delta_{n} z^{n}$ vanishes at $z^{n}=-1$. The proof of the theorem is complete. 
St. Ruscheweyh [15] showed that if $S_{\alpha}^{*}$ denotes the starlike functions of order $\alpha$ $\left(\operatorname{Re}\left\{z f^{\prime} / f\right\}>\alpha\right)$, then there is no value of $\delta>0$ such that $N_{\delta}\left(S_{\alpha}^{*}\right) \subset S^{*}$ for any $0 \leqslant \alpha<1$. On the other hand R. Fournier [2] showed that if $f(z)=z+a_{n+1} z^{n+1}$ $+\cdots \in T_{\alpha}$, where $T_{\alpha}$ is the subclass of $T$ defined by $\left|z f^{\prime}(z) / f(z)-1\right|<\alpha$, then $N_{\delta_{n}}(g) \subset T$, where $\delta_{n}=(1-\alpha) e^{-\alpha / n}$. He accounts for the differences by noting that the boundaries in Ruscheweyh's case, $\{w: \operatorname{Re} w>0\}$ and $\{w: \operatorname{Re} w>\alpha\}$, are not disjoint whereas in his case the boundaries, $\{w:|w-1|<1\}$ and $\{w:|w-1|<\alpha\}$, are. In our next result we show precisely how the boundaries affect the value of $\delta_{n}$ in situations more general than those mentioned above while at the same time including the results in [2, Theorems 2 and 3 and 5, Theorem 3(ii)].

Let $F$ and $G$ be fixed functions analytic and univalent in $\mathbf{D}$ with $F \prec G$ and $F(0)=G(0)=1$. It is known [1, p. 50] that $F$ and $G$ belong to $H^{p}$ for all $0<p<\frac{1}{2}$ and hence have radial limits almost everywhere. Let $E^{*}$ denote the set of all $\theta \in[0,2 \pi)$ such that $G\left(e^{i \theta}\right)$ exists. We next suppose that $G$ has the property that there exists an integer $N \geqslant 2$ such that

$$
\lambda \equiv \inf _{\theta \in E^{*}}\left|N-G\left(e^{i \theta}\right)\right|>0 .
$$

Define the classes $\mathscr{F}$ and $\mathscr{G}$ by

$$
\mathscr{F}=\left\{f \in \mathscr{H}: \frac{z f^{\prime}}{f} \prec F\right\} \quad \text { and } \quad \mathscr{G}=\left\{f \in \mathscr{H}: \frac{z f^{\prime}}{f} \prec G\right\} .
$$

Then we have the following result:

THEOREM 2. Let $\eta=\operatorname{dist}(F(\mathbf{D}), G(\mathbf{D}))$.

(a) If $\eta \neq 0$ and $g(z)=z+b_{n+1} z^{n+1}+\cdots \in \mathscr{F}$, then $N_{\delta_{n}}(g) \subset \mathscr{G}$, with

$$
\delta_{n}=\eta \exp \left[\int_{0}^{1} \frac{1}{t}\left\{\min _{|z|=t} \operatorname{Re} F\left(z^{n}\right)-1\right\} d t\right] .
$$

(b) If $\eta=0$ and if $G$ satisfies (11), then there exists a function $g_{N} \in \mathscr{F}_{\text {such }}$ that for no value of $\delta>0$ is $N_{\delta}\left(g_{N}\right) \subset \mathscr{G}$.

Proof. (a) If $g(z)=z+b_{n+1} z^{n+1}+\cdots \in \mathscr{F}$, it follows that $z g^{\prime} / g=F(\omega(z))$, where $\omega(z)=z^{n} \phi(z)$ and $|\phi(z)| \leqslant 1$. Hence we see that

$$
\begin{aligned}
r \frac{\partial}{\partial r} \operatorname{Re}\left\{\log \frac{g\left(r e^{i \theta}\right)}{r}\right\} & =\operatorname{Re}\left\{\frac{z g^{\prime}}{g}-1\right\} \geqslant \min _{|z|=r} \operatorname{Re} F(\omega(z))-1 \\
& \geqslant \min _{|z|=r} \operatorname{Re} F\left(z^{n}\right)-1
\end{aligned}
$$

and we conclude that

$$
\left|\frac{g\left(r e^{i \theta}\right)}{r}\right| \geqslant \exp \int_{0}^{r} \frac{1}{t}\left\{\min _{|z|=t} \operatorname{Re} F\left(z^{n}\right)-1\right\} d t .
$$

Since $G$ is univalent we see that $g \in \mathscr{G}$ if and only if $g(0)=0, g^{\prime}(0) \neq 0$ and

$$
\frac{z g^{\prime}}{g} \neq G\left(e^{i \theta}\right) \quad \text { for all } \theta \in E^{*}, z \in \mathbf{D} \text {. }
$$

It is evident that (13) holds only if $\left(k_{\theta} * g\right) / z \neq 0$ for all $\theta \in E^{*}$ and $z \in \mathbf{D}$, where

$$
k_{\theta}(z)=\frac{z}{(1-z)^{2}}-\frac{G\left(e^{i \theta}\right) z}{1-z} \text {. }
$$


We easily see that for $g(z)=z+b_{n+1} z^{n+1}+\cdots \in \mathscr{F}$ the range of values of $z g^{\prime} / g$ are contained in $F(\mathbf{D})$ and hence

$$
\left|\frac{k_{\theta} * g}{z}\right|=\left|\frac{g(z)}{z}\right|\left|\frac{z g^{\prime}(z)}{g(z)}-G\left(e^{i \theta}\right)\right| \geqslant \eta\left|\frac{g(z)}{z}\right| .
$$

In view of (12) we have

$$
\left|\frac{k_{\theta} * g}{z}\right| \geqslant \eta \cdot \exp \left[\int_{0}^{r} \frac{1}{t}\left\{\min _{|z|=t} \operatorname{Re} F\left(z^{n}\right)-1\right\} d t\right] .
$$

Since $F(0)=1$ we see that $\min _{|z|=t} \operatorname{Re} F\left(z^{n}\right) \leqslant 1,0<t<1$, and hence the righthand side of the last inequality is a decreasing function of $r$. This proves (a).

(b) Let $\lambda$ be given by (11) and define $g_{N}$ by

$$
g_{N}(z)=z \exp \left[\int_{0}^{z} \frac{1}{t}\left\{F\left(t^{n}\right)-1\right\} d t\right] .
$$

It is clear that $z g_{N}^{\prime}(z) / g_{N}(z)=F\left(z^{N}\right) \prec F(z)$ and hence $g_{N} \in \mathscr{F}$. Furthermore, since $\eta=0$, we have by (14)

$$
\inf \left|\frac{g_{N} * k_{\theta}}{z}\right|=\inf \left[\left|\frac{g_{N}(z)}{z}\right|\left|F\left(z^{N}\right)-G\left(e^{i \theta}\right)\right|\right]=0,
$$

where the infimum is taken over all $\theta \in E^{*}$ and $z \in \mathbf{D}$.

Assume there exists a $\delta>0$ such that $N_{\delta}\left(g_{N}\right) \subset \mathscr{G}$. Since $g_{N}^{\prime}(0) \neq 0$ we have $g_{N}(z) / z \neq 0$ in $|z| \leqslant r_{0}$ for some $0<r_{0}<1$. Clearly $\left|F\left(z^{N}\right)-G\left(e^{i \theta}\right)\right| \neq 0$ in $|z| \leqslant$ $r_{0}$. Hence for any fixed $\theta \in E^{*}$ we can conclude that $\left|g_{N} * k_{\theta} / z\right| \geqslant \varepsilon>0$ for some $\varepsilon$, when $|z| \leqslant r_{0}$. In view of (15), we can then select $z_{0} \in \mathbf{D}$, with $r_{0} \leqslant\left|z_{0}\right|<1$, and $\theta_{0} \in E^{*}$ such that

$$
\left|\frac{\left(g_{N} * k_{\theta_{0}}\right)\left(z_{0}\right)}{z_{0}}\right|<\frac{\lambda \delta r_{0}^{N-1}}{N} .
$$

Putting

$$
\mu=\frac{N}{\lambda} \frac{\left(g_{N} * k_{\theta_{0}}\right)\left(z_{0}\right)}{z_{0}^{N}}
$$

we observe that

$$
\frac{\left(g_{N} * k_{\theta_{0}}\right)\left(z_{0}\right)}{z_{0}}=\frac{\lambda \mu z_{0}^{N-1}}{N}
$$

and $|\mu|<\delta$. Let $f(z)=g_{N}(z)-\lambda \mu z^{N} / N C_{N}$, where $C_{N}=N-G\left(e^{i \theta_{0}}\right)$ is the $N$ th coefficient of $k_{\theta_{0}}(z)$. Now we see that since $f^{\prime}(z)-g_{N}^{\prime}(z)=-\lambda \mu z^{N-1} / C_{N}$ and $\left|\lambda / C_{N}\right| \leqslant 1$ we have $f \in N_{\delta}\left(g_{N}\right) \subset \mathscr{G}$. Finally, we have by (16)

$$
\frac{\left(k_{\theta_{0}} * f\right)\left(z_{0}\right)}{z_{0}}=\frac{\left(k_{\theta_{0}} * g_{N}\right)\left(z_{0}\right)}{z_{0}}-\frac{\lambda \mu z_{0}^{N-1}}{N}=0 .
$$

Hence $\left(k_{\theta_{0}} * f\right)\left(z_{0}\right) / z_{0}=0$ and so $f \notin \mathscr{G}$. This is a contradiction. The proof of the theorem is complete.

The author wishes to thank the referee for some useful comments. 


\section{REFERENCES}

1. P. L. Duren, Theory of $H^{p}$ spaces, Academic Press, New York, 1970.

2. R. Fournier, A note on neighborhoods of univalent functions, Proc. Amer. Math. Soc. 87 (1983), $117-120$.

3. A. W. Goodman, Univalent functions and nonanlytic curves, Proc. Amer. Math. Soc. 8 (1957), 598-601.

4. W. Kaplan, Close-to-convex schlicht functions, Michigan Math. J. 1 (1952), 261-265.

5. St. Ruscheweyh, Neighborhoods of univalent functions, Proc. Amer. Math. Soc. 81 (1981), 521-527.

6. St. Ruscheweyh and T. Sheil-Small, Hadamard products for schlicht functions and the Pólya-Schoenberg conjecture, Comment. Math. Helv. 48 (1973), 119-135.

Department of Mathematics, Purdue University, West Lafayette, Indiana 47907 